\title{
Found in translation of mTOR signaling
}

\author{
John G Clohessy ${ }^{1,2,{ }^{*}}$, Markus Reschke ${ }^{1,2, *}$, Pier Paolo Pandolfi ${ }^{1}$ \\ ${ }^{I}$ Cancer Genetics Program, Beth Israel Deaconess Cancer Center, Departments of Medicine and Pathology, Beth Israel Deacon- \\ ess Medical Center, Harvard Medical School, Boston, MA 02215, USA \\ Cell Research (2012) 22:1315-1318. doi:10.1038/cr.2012.85; published online 29 May 2012
}

The mammalian target of rapamycin (mTOR) protein kinase regulates a wide variety of cellular processes, including protein synthesis, yet the downstream translational program under the control of mTOR is poorly understood. Two recent studies by Hsieh et al. and Thoreen et al. now start to address this issue, and uncover a subset of genes translationally regulated by oncogenic mTOR signaling that may contribute to tumorigenesis.

The mTOR kinase is generally accepted to be a master regulator of protein synthesis, and mTOR signaling is frequently deregulated in human cancer $[1,2]$. mTOR itself can assemble either with raptor or rictor to form two distinct signaling complexes: the mTOR complex 1 (mTORC1) and the mTOR complex 2 (mTORC2), respectively [1, 3]. It is well established that mTORC1can regulate protein translation through phosphorylation of both 4E-BP1 and p70S6K1/2 (S6K) (Figure 1) [2, 4]. When hypo-phosphorylated, 4E-BP1 binds to and sequesters eIF4E, thereby inhibiting translation initiation. Upon phosphorylation by mTORC1, 4E-BP1 releases eIF4E, allowing formation

*These two authors contributed equally to this work.

Correspondence: Pier Paolo Pandolfi Tel: 617-735-2121; Fax: 617-735-2120

E-mail: ppandolf@bidmc.harvard.edu of the translation initiation complex eIF4F, and promoting cap-mediated translation. At the same time, activation of S6K by mTORC1 leads to the phosphorylation of ribosomal protein $\mathrm{S} 6$ (RpS6), which is important for global translation and cell growth [5]. However, our mechanistic understanding of events downstream of 4E-BP/eIF4E and $\mathrm{S} 6 \mathrm{~K} / \mathrm{RpS} 6$ are currently limited, and it is unclear whether mTOR translationally regulates a distinct set of genes that may contribute to cancer initiation and/ or progression.

Now, two papers in Nature from Hsieh et al. and Thoreen et al. address this question using a recently developed methodology termed "ribosome profiling" to uncover specific gene expression networks that are translationally regulated by oncogenic mTOR in prostate cancer cell lines [6] and mouse embryonic fibroblast (MEF) cells [7]. Ribosome profiling has been pioneered in the Weissman laboratory at UCSF and allows for the quantitative assessment of ribosomal occupancy along specific mRNAs undergoing translation $[8,9]$. It has now been successfully applied to a number of different cellular models, and has become a potent tool in uncovering previously unappreciated regulation and complexity of mRNA translation and gene expression $[10,11]$. Applying this method, the authors examined acute inactivation of mTOR signaling using mTOR inhibitors. While Hsieh et $a l$. used both the allosteric inhibitor ra- pamycin and the mTOR kinase inhibitor PP242, Thoreen et al. used the mTOR kinase inhibitor Torin1. Although little changes were observed with rapamycin treatment (probably due to the inability of rapamycin to effectively block 4EBPs), acute treatment with the mTOR kinase inhibitors allowed for the evaluation of primary translational effects due to robust inhibition of mTOR. Unexpectedly, both manuscripts report that the translation of relatively few mRNAs (approximately 200) were specifically downregulated upon treatment with the mTOR inhibitors. It is also worth noting that Thoreen et al. identify a number of putative IRES-containing mRNAs and histone mRNAs to be translationally upregulated by Torin1. On the other hand, Hsieh et al. identify a small number of mRNA molecules to be upregulated by rapamycin treatment. Although not evaluated in these studies, understanding how these proteins mediate the output of mTOR signaling may further illuminate the role of mTOR in regulating distinct cellular processes.

Weissman's work has previously shown that one of the advantages of ribosome profiling is that the resulting delineation of ribosomal occupancy on mRNAs reveals alternate regulation of these transcripts, for example through the binding and use of upstream open reading frames (uORFs) or the utilization of ribosomal pausing on mRNA transcripts $[8,9]$. The work presented in these manuscripts do not elaborate on 


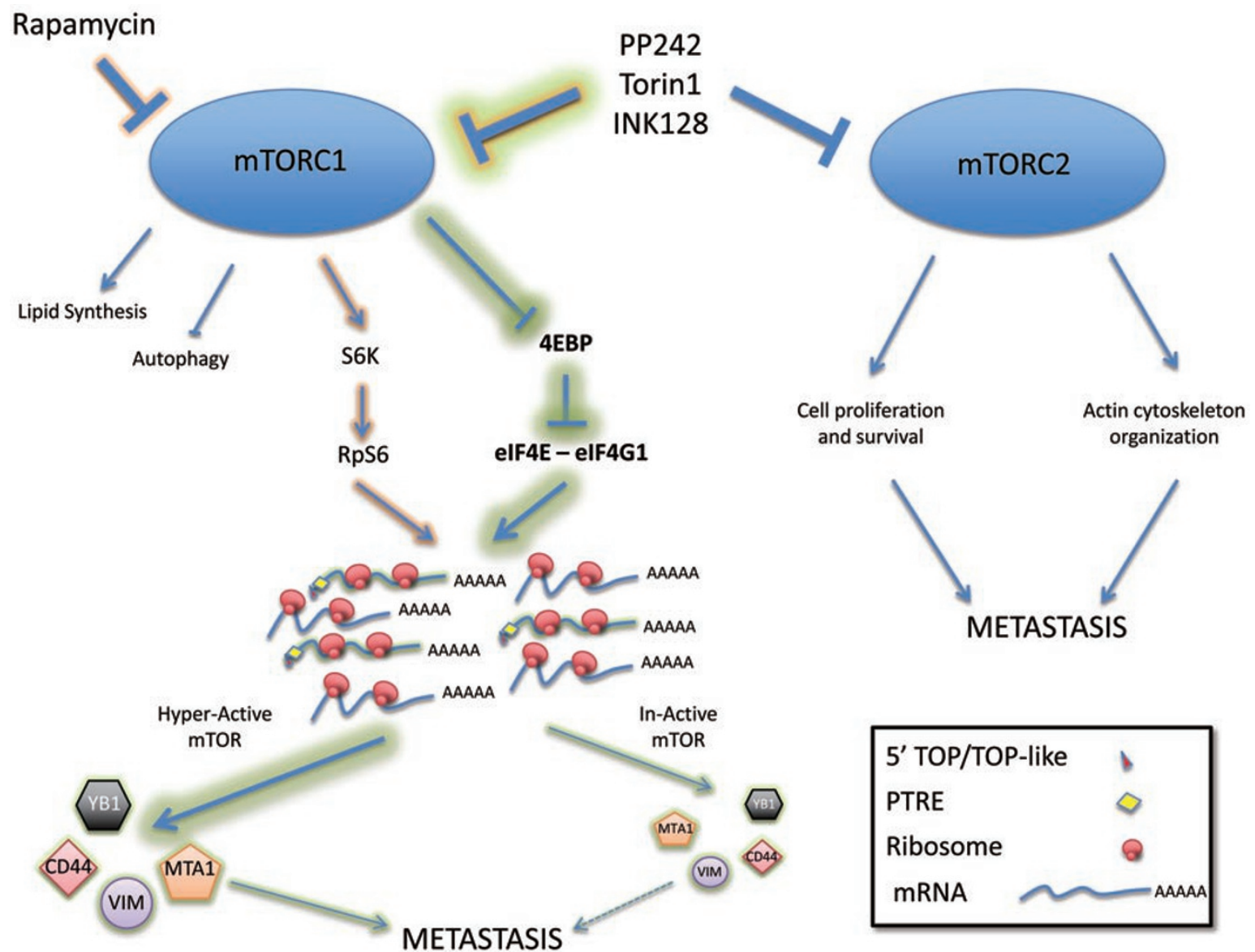

Figure 1 Translational impact of the mTOR signaling pathway. Multiple cellular pathways are regulated by signaling from mTOR complexes, including regulation of autophagy, lipid metabolism, and control of actin cytoskeleton in addition to the regulation of translation. Translational control by mTOR is regulated predominantly by signaling from mTORC1, and mediated by phosphorylation of 4E-BPs and S6K, which directly influence the function of elF4E and RpS6, respectively. Now, two papers by Hsieh et al. and Thoreen et al. demonstrate that mTOR signaling to the ribosome can regulate a specific subset of mRNA transcripts, the majority of which contain a $5^{\prime}$ TOP, a $5^{\prime}$ TOP-like or a novel PRTE element in their $5^{\prime}$ UTR regions. While Thoreen et al. now establish an important mTOR-sensitive regulation of elF4G1 downstream of 4E-BPs, Hsieh et al. focus on a subset of mRNA transcripts (YB-1, CD44, MTA1 and vimentin) strongly linked to regulation of metastasis, and propose that they play a role in the progression of Pten-loss-driven prostate cancer through hyperactivation of the mTOR signaling pathway. While, the ATP site inhibitor INK128 was shown to inhibit tumor progression in mice, it should be noted that such inhibitors also block all activities of $\mathrm{mTORC} 1$ and 2, and while they can successfully block the translational up-regulation of the 4-gene metastasis signature described by Hsieh et al., they also block other metastatic pathways regulated by independently controlled mTOR pathways.

such alterations to ribosomal occupancy in response to mTOR inhibition. Thus, it remains to be seen whether further probing of these datasets will uncover such a differential regulation.

For the genes found to be translationally downregulated upon mTOR inhibition, analysis of their $5^{\prime}$ UTRs allowed for the identification of novel regulatory elements that dictate sensitiv- ity to mTOR activity. Surprisingly, both studies suggest that mTOR-sensitive mRNAs do not contain complex and highly structured 5' UTRs as previously thought. In addition to known mTOR sensitive $5^{\prime}$ terminal oligopyrimidine tract ( $5^{\prime}$ TOP) containing mRNAs, Hsieh et al. identify enrichment of a pyrimidine-rich translational element (PRTE) that can be found in the $5^{\prime}$
UTR of many of the mRNAs identified (including the $5^{\prime}$ UTR of some $5^{\prime}$ TOPcontaining mRNAs), while Thoreen et al. identify a $5^{\prime}$ TOP-like sequence within the vicinity of the translational start site. Although known mTORsensitive mRNAs (e.g., p53 [12]) are not identified as part of this list, they may represent mTOR sensitive downstream transcripts, whose translation is 
regulated by primary targets identified in this study (e.g., RpL26 in the case of p53) as part of the gene set that is sensitive to mTOR inhibition.

Closer examination of this gene set revealed that these mRNAs can be categorized in a wide variety of cellular functions, with a preference for key components of the translational apparatus (including ribosomal proteins, elongation factors and initiation factors), confirming that mTOR can directly impact the translational capacity of highly proliferating cells (as is the case for many cancer cells). Notably, the second largest group of translationally downregulated genes as identified by Hsieh $e t$ $a l$. corresponded to known cell invasion and metastasis genes, indicating that mTOR may direct cancer progression. Specifically, the authors identify YB1, CD44, vimentin and MTA1 as key cell invasion and metastasis mRNAs that represent a distinct gene signature that is under translational regulation by mTOR. Indeed, modulation of any one of these four mRNAs impacted cancer cell migration in vitro [6].

Both studies now clearly demonstrate that, at least in an acute setting, the strong translational impact of mTOR inactivation on translation is driven by 4E-BP proteins. While Hsieh et al. do not investigate further the mechanistic properties that underlie the control of translational initiation, Thoreen et al. clearly demonstrate that activation of 4E-BPs disrupt the interaction between eIF4E and eIF4G1. Thoreen $e t$ al. demonstrate that the regulation of this interaction plays a critical role in coordinating translation of $5^{\prime} \mathrm{TOP}$ and $5^{\prime}$ TOP-like mRNAs as eIF4G1 depletion selectively represses TOP mRNA translation, in a manner that is downstream of 4E-BP proteins.

On the other hand, Hsieh et al. made use of a Pten-loss-driven mouse model of prostate cancer, in order to validate their findings in vivo in the context of mTOR hyperactivation [13]. Conditional inactivation of Pten in the mouse prostate, using Cre recombinase driven by the androgen-sensitive Probasin $(\mathrm{Pb})$ promoter, is a well characterized and faithful model of human prostate cancer [14-16]. The authors confirmed that their 4-gene signature is expressed both in basal (CK5+) and luminal (CK8+) Pten-deficient mouse prostate cells, and further examined how cancer cell metastasis was impacted by blocking mTOR activity in the context of Ptenloss-driven prostate cancer. Although the issue of metastasis in mouse models of prostate cancer driven by Pten loss is a hotly debated topic amongst mouse geneticists, the Pten conditional model used by Hsieh et al. is reported to show metastasis to the lymph nodes [17]. Metastatic lesions in these mice demonstrated robust expression of YB1 and the prostate-specific androgen receptor, which mirrors strong expression of YB1 in human prostate cancer samples. Furthermore, treatment with the novel INK128 ATP site inhibitor appeared to limit the invasive progression of the prostate tumors in mice, and also impacted the number and size of lymph node metastasis. Thus, inhibition of mTOR using ATP-kinase inhibitors of the protein holds promise as a method of targeting the multiple contributions of mTOR to tumorigenesis.

It is important to note that the genetic ablation of mTOR in a Pten-loss driven mouse model of prostate cancer has already been demonstrated to block prostate cancer development [18]. Similarly, as with genetic inactivation of mTOR, the INK128 inhibitor blocks all activities of mTOR, including those of mTORC2 (Figure 1), which are already known to regulate EMT, motility, and metastasis of colorectal cancer cells via RhoA and Rac1 [19]. Thus, the physiological relevance of mTOR's ability to regulate metastasis through translational control of this gene set remains to be fully determined in vivo. While, eIF4E with reduced activity has been previously shown to restrict Ptenloss-driven prostate cancer in vivo [20], it is also important to clarify whether mTOR sensitive mRNAs are specifically altered in this setting, and how the interaction with eIF4G1 is impacted by this mutation. The development of mouse models that allow for the inducible expression of a phospho-deficient 4E-BP1 mutant or for eIF4G1 loss of function, may help to better define the extent to which the transient inhibition of this arm of the pathway contributes to tumor suppression in mice.

In conclusion, these studies present an elegant analysis of the translational output in response to the inhibition of mTOR. The question remains as to what other elements help mTOR coordinate the translational regulation, and how translation is affected upon chronic mTOR activation or inhibition. For example, are other proteins involved in regulation of mTOR-sensitive genes at the ribosome and what of other modes of mTOR hyperactivation (e.g., Tsc1/2 loss) display similar translational signatures? Understanding and precisely identifying these key mTOR-responsive mediators of translational output may allow the characterization of the mechanisms by which a wide range of signaling pathways that converge on the ribosome regulate translation. Furthermore, this may foster the development of therapies that target tumor-specific translational regulators to ultimately eradicate cancer and metastasis.

\section{References}

1 Zoncu R, Efeyan A, Sabatini DM. mTOR: from growth signal integration to cancer, diabetes and ageing. Nat Rev Mol Cell Biol 2011; 12:21-35.

2 Ma XM, Blenis J. Molecular mechanisms of mTOR-mediated translational control. Nat Rev Mol Cell Biol 2009; 10:307-318.

3 Wullschleger S, Loewith R, Hall MN. TOR signaling in growth and metabolism. Cell 2006; 124:471-484.

4 Mamane Y, Petroulakis E, LeBacquer O, Sonenberg N. mTOR, translation initiation and cancer. Oncogene 2006; 25:6416-6422. 
5 Ruvinsky I, Sharon N, Lerer T, et al. Ribosomal protein S6 phosphorylation is a determinant of cell size and glucose homeostasis. Genes Dev 2005; 19:2199-2211.

6 Hsieh AC, Liu Y, Edlind MP, et al. The translational landscape of mTOR signalling steers cancer initiation and metastasis. Nature 2012; 485:55-61.

7 Thoreen CC, Chantranupong L, Keys HR, Wang T, Gray NS, Sabatini DM. A unifying model for mTORC1-mediated regulation of mRNA translation. $\mathrm{Na}$ ture 2012; 485:109-113.

8 Ingolia NT, Lareau LF, Weissman JS. Ribosome profiling of mouse embryonic stem cells reveals the complexity and dynamics of mammalian proteomes. Cell 2011; 147:789-802.

9 Ingolia NT, Ghaemmaghami S, Newman J R S, Weissman JS. Genomewide analysis in vivo of translation with nucleotide resolution using ribosome profiling. Science 2009; 324:218-223.

10 Brar GA, Yassour M, Friedman N, Regev A, Ingolia NT, Weissman JS. High- resolution view of the yeast meiotic program revealed by ribosome profiling. Science 2012; 335:552-557.

11 Guo H, Ingolia NT, Weissman JS, Bartel DP. Mammalian microRNAs predominantly act to decrease target mRNA levels. Nature 2010; 466:835840.

12 Alimonti A, Nardella C, Chen Z, et al. A novel type of cellular senescence that can be enhanced in mouse models and human tumor xenografts to suppress prostate tumorigenesis. J Clin Invest 2010; 120:681-693.

13 Salmena L, Carracedo A, Pandolfi PP. Tenets of PTEN tumor suppression. Cell 2008; 133:403-414.

14 Di Cristofano A, Pesce B, Cordon-Cardo C, Pandolfi PP. Pten is essential for embryonic development and tumour suppression. Nat Genet 1998; 19:348355.

15 Trotman LC, Niki M, Dotan ZA, et al. Pten dose dictates cancer progression in the prostate. PLoS Biol 2003; 1:E59.

16 Di Cristofano A, De Acetis M, Koff A,
Cordon-Cardo C, Pandolfi PP. Pten and p27KIP1 cooperate in prostate cancer tumor suppression in the mouse. Nat Genet 2001; 27:222-224.

17 Wang S, Gao J, Lei Q, et al. Prostatespecific deletion of the murine Pten tumor suppressor gene leads to metastatic prostate cancer. Cancer Cell 2003; 4:209-221.

18 Nardella C, Carracedo A, Alimonti $\mathrm{A}$, et al. Differential requirement of mTOR in postmitotic tissues and tumorigenesis. Sci Signal 2009; 2 :ra2.

19 Gulhati P, Bowen KA, Liu J, et al. mTORC1 and mTORC2 regulate EMT, motility, and metastasis of colorectal cancer via RhoA and Rac1 signaling pathways. Cancer Res 2011; 71:32463256.

20 Furic L, Rong L, Larsson O, et al. eIF4E phosphorylation promotes tumorigenesis and is associated with prostate cancer progression. Proc Natl Acad Sci USA 2010; 107:14134-14139. 